\title{
PP05
}

\section{HANGE IN THE APPEARANCE OF OBJECTS ACCORDING TO THE RATIO OF DIRECT AND DIFFUSIVE LIGHT}

\author{
Yoko Mizokami et al.
}

DOI 10.25039/x46.2019.PP05

from

CIE x046:2019

\section{Proceedings}

of the

29th CIE SESSION

Washington D.C., USA, June 14 - 22, 2019

(DOI 10.25039/x46.2019)

The paper has been presented at the 29th CIE Session, Washington D.C., USA, June 14-22, 2019. It has not been peer-reviewed by CIE.

(C) CIE 2019

All rights reserved. Unless otherwise specified, no part of this publication may be reproduced or utilized in any form or by any means, electronic or mechanical, including photocopying and microfilm, without permission in writing from CIE Central Bureau at the address below. Any mention of organizations or products does not imply endorsement by the CIE.

This paper is made available open access for individual use. However, in all other cases all rights are reserved unless explicit permission is sought from and given by the CIE.

CIE Central Bureau

Babenbergerstrasse 9

A-1010 Vienna

Austria

Tel.: +4317143187

e-mail: ciecb@cie.co.at

www.cie.co.at 


\title{
CHANGE IN THE APPEARANCE OF OBJECTS ACCORDING TO THE RATIO OF DIRECT AND DIFFUSIVE LIGHT
}

\author{
Mizokami, Y.. ${ }^{1}$, Kiyasu, Y. ${ }^{1}$, Yaguchi, H. ${ }^{1}$ \\ ${ }^{1}$ Chiba University, Chiba, JAPAN \\ mizokami@faculty.chiba-u.jp
}

DOI 10.25039/x46.2019.PP05

\begin{abstract}
We investigated how the appearance of an object surface changed according to the ratio of diffusive and direct light components. A miniature room was illuminated by mixed lighting with directional light and diffusive light. We evaluated the glossiness and roughness of test samples under five diffuseness levels. We used glossy and matte square samples with a sine-wavesurface which depth was 1 and $0.5 \mathrm{~mm}$, and three types of frequencies. Our results show that samples with high frequency and rough surface tend to appear less glossy and smoother as the diffuseness of light increase. Glossy samples appear less glossy and matte samples appear smoother under diffusive light. It was suggested that the properties of luminance distribution on samples would contribute to the object appearance. Further analysis suggested a possibility to predict the glossiness and roughness based on both image statistics and the physical factors of samples.
\end{abstract}

Keywords: Surface appearance, Diffuseness of illumination

\section{Introduction}

Lighting environment has become diverse due to the improvement of solid-state lighting technology, and it is critical to consider the appearance of objects under various lighting conditions. Shape, texture, and light are crucial factors to determine the appearance of an object. However, it had not been systematically analysed how surface appearance is influenced by the diffuseness of lighting. Some researchers investigated the surface property of objects and lighting direction (e.g. PONT 2007; XIA 2017). Our previous studies showed that the diffuseness of lighting mainly influenced the appearance of the glossiness and the roughness of an object (MIZOKAMI 2016 2017; KIYASU 2017). We controlled the diffuseness of by changing the position of a diffuser board under a lamp in the previous studies.

However, it was not investigated systematically how the combination of direct light and diffusive light influences the appearance of an object (KIYASU 2018). In our daily life, we often see lighting environments consists of a combination of direct light and diffusive light. Thus, it is essential to clarify their effect on the appearance of objects. In this study, we investigate how the appearance of the object surface, especially glossiness and roughness changes according to the ratio of diffusive and direct light components.

\section{Experiment}

\subsection{Environment}

As shown in Figure 1, we placed a miniature room in a dark room. The miniature room was illuminated by mixed lighting with directional light and diffusive light. We used two LED lamps and a diffuser board. One was far from the diffuser board, giving a diffusive lighting condition. The other was close to the diffuser boards, giving a direct lighting condition. A test sample for evaluations was placed at the center of the miniature room. A luminance level at the test sample plane was $60 \mathrm{~cd} / \mathrm{m}^{2}$ when a white calibration plate was placed and it was kept constant among all lighting conditions. A black square paper was put on the back wall to avoid the influence of simultaneous brightness contrast. 


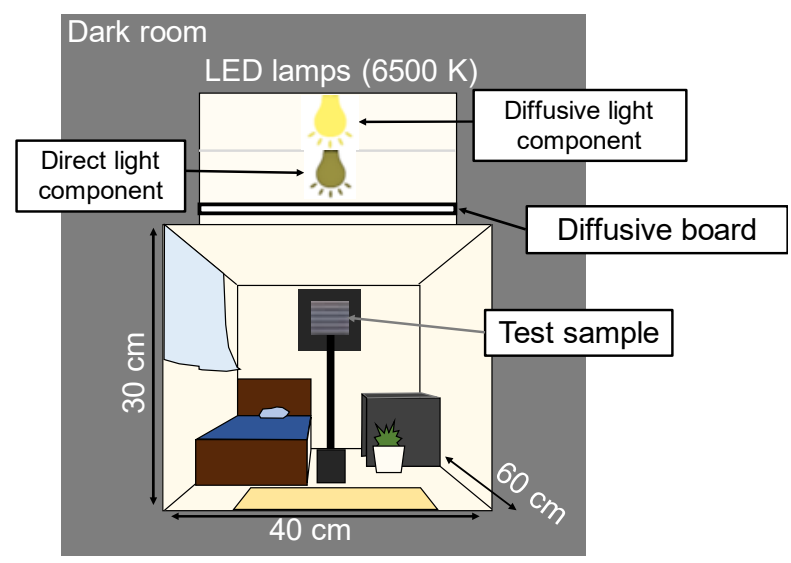

Figure 1 - Front View of a Miniature Room

We defined the diffuseness of the lighting by based on Cuttle's vector/scalar illumination ratio (CUTTLE 2013; XIA 2017). We measured the diffuseness of the illumination at the position of a stimulus based on a cubic illuminance measurement. The illuminance of six directions was measured to calculate vector illuminance $(|\mathrm{E}|)$ and scalar illuminance $\left(E_{\mathrm{sr}}\right)$. Diffuseness $\left(D_{\text {cuttle }}\right.$ $\left.=1-\left(|E| / E_{s r}\right) / 4\right)$ can be specified from cylindrical illuminance and the working plane illuminance. $D_{\text {cuttle }}=0$ indicates a direct light and $D$ cuttle $=1$ indicates a diffusive light. We tested the five diffuseness levels of lighting by changing the intensity ratio of each lamp: $0,38,0,44,0,47,0,53$, and 0,54 .

Figure 2 shows test samples for evaluation under each level of light diffuseness. Test samples were $3 \mathrm{~cm}$-square resin patches made with a 3D printer. The samples had a sine-wave-surface which the depth of the wave was 1 and $0,5 \mathrm{~mm}$, and three types of frequencies $(3,6$, and 9 waves per sample). We prepared matte, and gloss samples which glossiness measured by a gloss meter were 0,6 and 16 , respectively.

Plane samples with sine wave surface $\left(3 \times 3^{\circ}\right)$

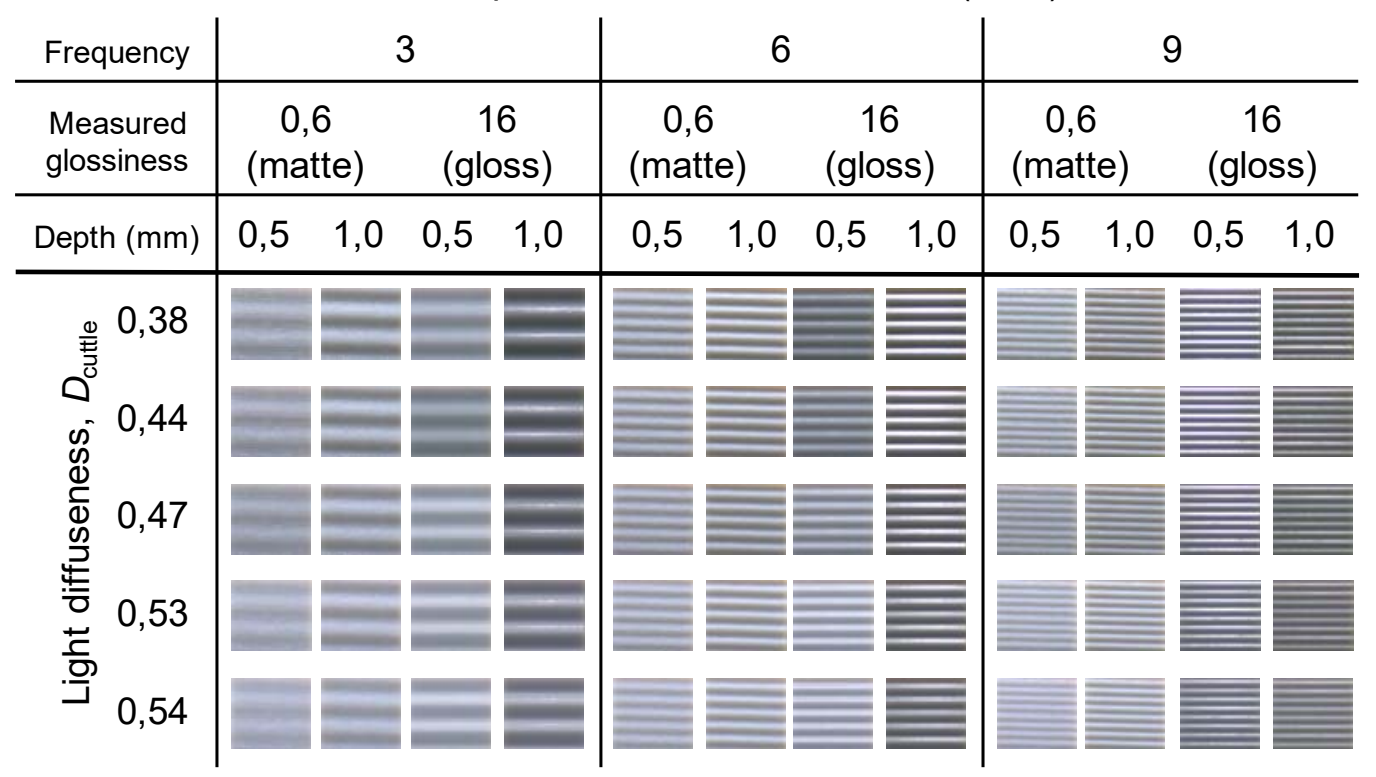

Figure 2 - Test Samples under Each Lighting Condition 


\subsection{Procedure}

An observer adapted a lighting condition for a minute and evaluated "Glossiness" and "Roughness" of the surface of test samples using a Visual Analogue Scale (VAS). After they evaluated 12 test samples, the light diffuseness condition was changed. They repeated the same task for all five lighting conditions in random order. The observers made evaluations three times each for all conditions. Nine observers participated.

\section{Results and Discussion}

Figure 3 shows the average results of all observers. An error bar shows the standard deviation. The abscissa shows the diffuseness levels defined by Cuttle's method. The ordinate shows the evaluation value. Samples tended to appear less glossy and smoother as the lighting became more diffusive. Especially, surfaces in high frequency and $1 \mathrm{~mm}$ conditions were strongly influenced by the diffuseness levels of lighting. Perceived glossiness for glossy and highfrequency-surface samples showed a larger influence of the diffuseness levels of lighting. Perceived roughness for matte surface samples showed the clearer influence of the diffuseness levels.
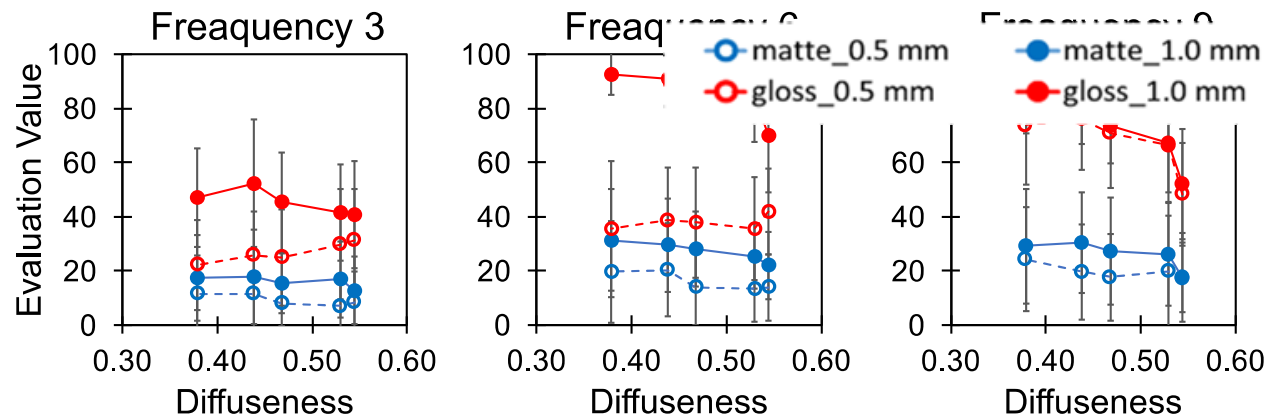

(a) Glossiness
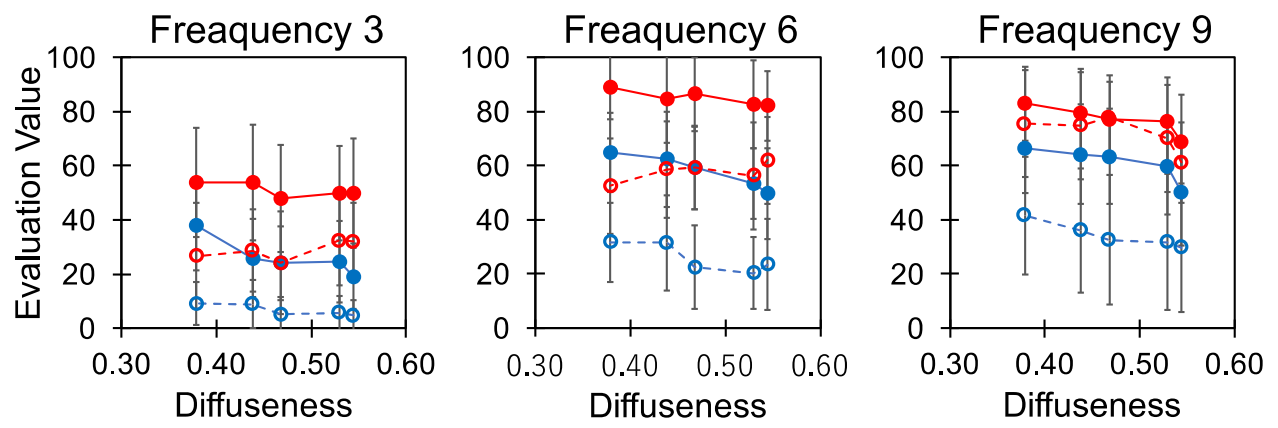

(b) Roughness

Figure 3 - Average Results of All Observers: (a) Glossiness, (b) Roughness.

We analyzed the relationship between the appearance of objects and the luminance histogram of samples. Some examples are shown in Figure 4. The glossiness evaluation showed high correlations with the standard deviation $(r=0,9)$, contrast $(r=0,9)$, and skewness $(r=0,8)$ of the luminance histogram. Roughness evaluation showed high correlations with the contrast $(r$ $=0,9)$ and the standard deviation $(r=0,9)$, and a rather moderate correlation with the skewness $(r=0,7)$. These results suggest that the appearance of objects is changed with the characteristics of the luminance distribution of samples. 

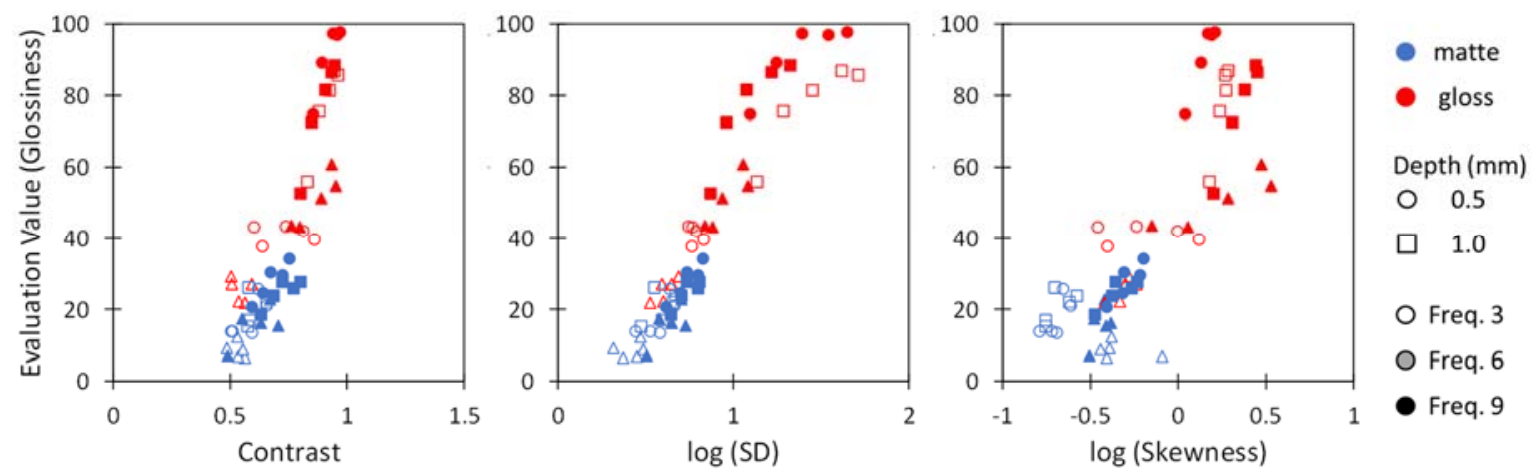

(a) Glossiness
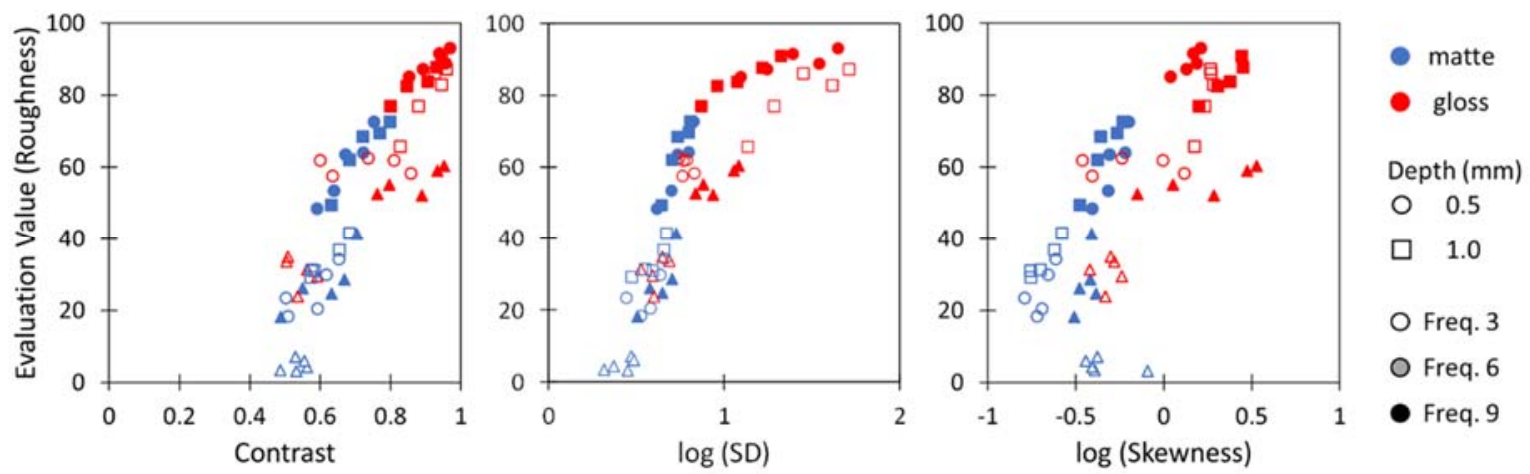

(b) Roughness

Figure 4 - Correlation of Evaluation Values with Contrast, Standard Deviation (SD), and Skewness of Luminance Histogram of Test Samples: (a) Glossiness, (b) Roughness.

It has been suggested that the image statistics such as the skewness of luminance and the distribution of highlights contribute a surface appearance (MOTOYOSHI 2007; MALOW 2013). We further examined the factors of image statistics that affected the appearance of objects using a multiple regression analysis where standard deviation, skewness, luminance gradient, the ratio of highlight region, and full width half maximum in the luminance distribution of test samples were set as independent variables while the glossiness or roughness evaluation served as a dependent variable. As shown in Figure $5(\mathrm{a})$, Equations 1 and 2, the results show high correlation ( $r^{2}=0,93$ for glossiness, $r^{2}=0,86$ for roughness). This suggests that the glossiness and roughness evaluation can be predicted by image statistics. However, the fitting to the roughness evaluation was worse than the glossiness evaluation, implying that image statistics may not be enough to predict roughness appearance and other factors such as threedimensional cues and a roughness constancy compensating change in roughness appearance due to the lighting diffuseness.

$$
\begin{aligned}
& \text { Glossiness }=-0,98+67,54 \log (S D)+6,91 \log (\text { LGrad })-16,04 \log (\text { FWHM })-73,46 \text { RHigh } \\
& \text { Roughness }=-66.56+114,01 \log (S D)+27,34 \log (F W H M)-218,62 \text { RHigh }
\end{aligned}
$$

where

Glossiness is the glossiness evaluation;

Roughness is the roughness evaluation;

$S D \quad$ is the standard deviation;

LGrad is luminance gradient;

FWHM is full width half maximum;

RHigh is the ratio of highlight region. 
We also examined the physical factors that have affected the appearance of objects using a multiple regression analysis where the measured glossiness, the depth of sine wave, and lighting diffuseness were independent variables, and the glossiness or roughness evaluation was a dependent variable. As shown in Figure 5 (b), Equations 3 and 4, the results also show high correlation $\left(r^{2}=0,80\right.$. for glossiness, $r^{2}=0,88$ for roughness). The glossiness and roughness evaluation can be predicted by physical measurement values also.

$$
\begin{aligned}
& \text { Glossiness }=-3,35-51.47 \mathrm{D}_{\text {cuttle }}+237,7 \text { MGls }+31,64 \text { Depth }+32,41 \mathrm{Frq} \\
& \text { Roughness }=-9,33-47,36 D_{\text {cuttle }}+172,64 \text { MGls }+44,27 \text { Depth }+49,19 \mathrm{Frq}
\end{aligned}
$$

where

$D_{\text {Cuttle }}$ is the diffuseness;

MGls is the measured glossiness;

Depth is the depth of sine-wave on the sample surface;

$\mathrm{Frq}$ is the frequency of sine-wave on the sample surface.
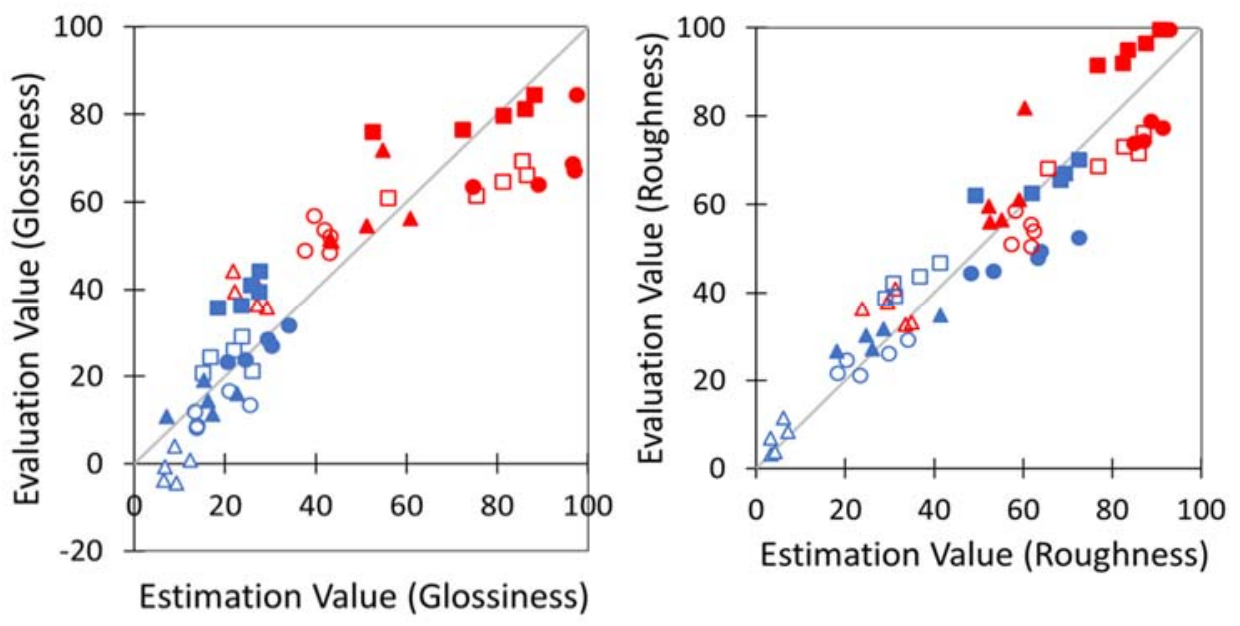

(a) Image Statistics Factors
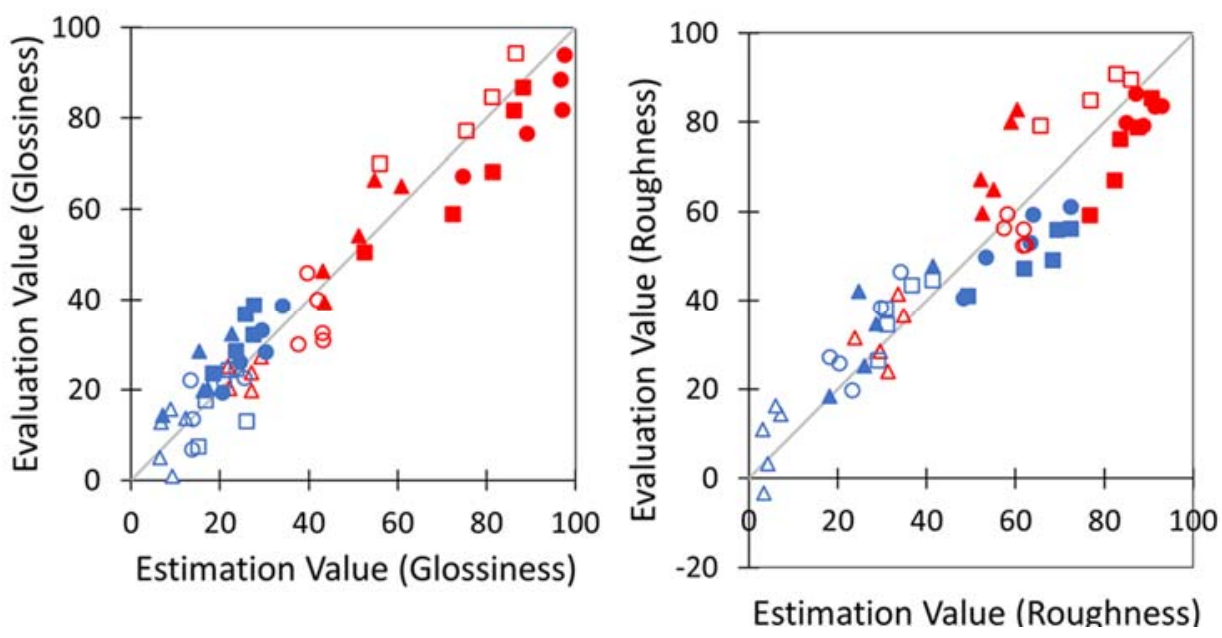

- matte

gloss

Depth (mm)

○ 0.5

1.0

O Freq. 3

O Freq. 6

Freq. 9

(b) Physical Factors

Figure 5 - The Result of Multiple Regression Analysis: (a) based on Statistical Factors of Luminance Distribution, (b) based on Physical Factors.

It should be noted that the equations shown here are based on the data in limited lighting conditions and samples and we need further investigations to evaluate the validity of those predictions and to generalize them to a wider range of lighting conditions and objects. However, 
they suggest that the surface appearance of objects under the mixture of diffusive and direct light with various diffuseness would be predicted by image statistics and/or physical measurement values.

\section{Conclusion}

We investigated how the glossiness and roughness appearance of object surface changed according to the ratio of diffusive and direct light components using glossy and matte square samples with a sine-wave surface which depth was 1 and $0.5 \mathrm{~mm}$, and three types of frequencies. Our results show that samples with high frequency and rough surface tend to appear less glossy and smoother as the diffuseness of light increase. Glossy samples appear less glossy and matte samples appear smoother under diffusive light condition. It was suggested that the change in luminance histogram and distribution (standard deviation, contrast, and skewness) would contribute to the appearance of an object. It was also suggested the possibility to predict the glossiness and roughness based on both image statistics and the physical factors of samples. It is necessary to consider the shape and characteristics of an object surface when predicting the appearance under variations of lighting diffuseness.

\section{Acknowledgement}

Supported by JSPS KAKENHI 16 K00368 and the Konica Minolta Imaging Science Encouragement Award.

\section{References}

CUTTLE, C. 2013. Research Note: A practical approach to cubic illuminance measurement, Lighting Research \& Technology, 46, 31-34.

KIYASU, Y., MIZOKAMI, Y., YAGUCHI, Y. 2017. Influence of changes in diffusivity of lighting on appearance of object surface, $24^{\text {th }}$ International Colour Vision Society, Abstract book, 77.

KIYASU, Y., MIZOKAMI, Y., YAGUCHI, Y. 2018. How direct light and diffusive light influence the appearance of objects? OSA Fall Vision Meeting, Abstract book.

MALOW, P. J., ANDERSON, B. L. 2013. Generative constraints on image cues for perceived gloss. Journal of Vision, 13(14)2, 1-23.

MIZOKAMI, Y., NABAE, Y., YAGUCHI, Y. 2016. Influence of diffusibility of illumination on the impression of surface appearance, Perception 55(45), 136-137.

MIZOKAMI, Y., KIYASU, Y., YAGUCHI, Y. 2017. Appearance of surface property influenced by the diffuseness of lighting, Vision Sciences Society 2017 Annual Meeting, Journal of Vision, 17.

MOTOYOSHI, I., NISHIDA, S., SHARAN, L., ADELSON. E. H. 2007. Image statistics and the perception of surface qualities, Nature, 447, 206-209.

PONT, S. C., KOENDERINK, J. J. 2007. Matching illumination of solid objects. Perception \& Psychophysics, 69(3), 459-468.

XIA, L., PONT, S. C., HEYNDERICKX, I. 2017. Light diffuseness metric, Part 1: Theory. Lighting Research \& Technology, 49(4), 411-427.

XIA, L., PONT, S. C., HEYNDERICKX, I. 2017. Light diffuseness metric, Part 2: Describing, measuring and visualising the light flow and diffuseness in three-dimensional spaces. Lighting Research \& Technology, 49(4), 428-445. 